\title{
Molecular/ Genetic/ Protein Characterizing of Bladder Cancer Circulating Tumor Cells
}

\author{
Nicolas Seranio $^{\mathrm{a}}$, S. Bruce Malkowicz ${ }^{\mathrm{b}}$, John Christodouleas ${ }^{\mathrm{c}}$ and Gary D. Kao ${ }^{\mathrm{c}, *}$ \\ ${ }^{a}$ Department of Urology (presently resident physician, Stanford Department of Urology), University of \\ Pennsylvania School of Medicine, Pennsylvania, PA, USA \\ ${ }^{\mathrm{b}}$ Department of Urology, University of Pennsylvania School of Medicine, Pennsylvania, PA, USA \\ ${ }^{\mathrm{c}}$ Department of Radiation Oncology, University of Pennsylvania School of Medicine, Pennsylvania, PA, USA
}

\begin{abstract}
.
Background: Circulating tumor cells (CTCs) assays are a promising noninvasive way to interrogate the status of disease in bladder cancer. Since the molecular signature of cancer cells may dictate their susceptibility to targeted or immunotherapy, the full value of CTC assays may be in such subsequent characterization. These secondary features may help increasingly guide future clinical management of bladder cancer.

Objective: This review aims to survey advances in the secondary molecular characterization of CTCs in bladder cancer.

Methods: Using PRISMA guidelines, a systematic search of PubMed articles published between January 1990 to February 2019 was conducted to identify bladder CTCs studies that included protein, gene expression, or molecular characterization data.

Results: Three original articles were identified. These articles assessed either PD-L1, HER2, or STn.

Conclusions: Our systematic review reveals a relative paucity of studies that undertake molecular or genetic characterization of CTCs in bladder cancer. Studies of that identify prognostic markers in bladder cancer will become increasingly useful as targeted and immunotherapy become integrated into the management of bladder cancer.
\end{abstract}

\section{HIGHLIGHTS}

- Characterization of bladder cancer CTCs can potentially inform subsequent clinical management

- PD-L1 and HER2 expression on CTCs may guide patient selection for targeted therapies.

- Sialyl-Tn (STn) expression by CTCs may be associated with increased aggressiveness and a worse prognosis.

\footnotetext{
*Correspondence to: Gary D. Kao, Department of Radiation Oncology, University of Pennsylvania School of Medicine, Pennsylvania, PA, USA. E-mail: Gary.Kao@pennmedicine. upenn.edu.
}

\section{INTRODUCTION}

Bladder cancer is one of the most common malignancies with 81,180 newly diagnosed cases and over 17,240 deaths predicted to occur in the United States in 2018 [1]. Standard methods for diagnosis and managing bladder cancer include cytological evaluation of urine, radiographic imaging, and cystoscopy [2]. There is a substantial interest in testing whether circulating tumor cells (CTCs) may complement conventional methods for guiding bladder cancer management.

CTCs are tumor cells that have shed into the vasculature or lymphatics from a primary tumor, and 
are carried around the body in the blood [3]. Detecting the presence of CTCs in the peripheral blood is a potentially less invasive way to identify advanced and metastatic disease earlier. In recent years there has been a surge in CTC technologies with over fifty assays for detection and enrichment that have already been described [4].

While there remains much experimental interest in the area of CTCs no CTC assay as yet been incorporated into the standard of care for any types of cancers. Studies have assessed whether the presence of CTCs in the peripheral blood may be an independent predictive indicator of poor outcomes for patients [5]. For example, in breast cancer it was shown that the number of CTC is an independent prognostic factor for progression-free survival (PFS) and overall survival (OS) [6]. Measuring CTC levels in small-cell lung cancer patients at base line showed that patients with CTC levels above 50 per $7.5 \mathrm{~mL}$ of blood were significantly associated with a higher stage and the presence of liver metastasis [7]. A recently published metaanalysis indicated that in colorectal cancer patients treated with chemotherapy, high numbers of CTCs correlate with poor OS [8].

As many cancer patients may already be receiving maximal conventional therapy, simple CTC counts may be insufficient to change therapy In contrast, monitoring of the CTC genomic and epigenomic profile might also better inform treatment selection and usefully suggest the need for innovative treatment. In metastatic breast cancer the presence of ESR1 methylation in CTCs from serial blood samples was shown to be associated with lack of response to everolimus/exemestane. This approach coupled with the emergence of targeted immunotherapy can lead to powerful and tailored treatments [9-12]. Preliminary evidence suggests that longitudinal on-treatment monitoring of CTCs, ctDNA, or PD-L1 dynamics might be used as a marker of response to identify the patients more likely to benefit from immunotherapy, thus sparing nonresponding patients from such treatment. Blood-based monitoring may also help decide whether to continue immunotherapy or not in cases of pseudoprogression.

This systematic review was conducted to survey CTC assays related to bladder cancer that have been published in the past decade. Only studies that went beyond simply identifying or counting of bladder circulating tumor cells, and examined the molecular, genetic, or protein expression patterns of such cells were selected. These few studies illustrate what may lie ahead for the field.

\section{METHODS}

\section{Search strategy}

The Preferred Reporting Items for Systematic Reviews and Meta-analysis (PRISMA) framework was used for this review. The search attempted to identify all articles which contained molecular characterization of CTCs with the potential to guide management. A systematic review of the scientific literature was performed using Medline/ PubMed on the 20th of February 2019. The following keywords were included in the search: "bladder cancer", "urothelial carcinoma", "circulating tumor cell", and "CTC". There were no restrictions placed on language or date of publication.

\section{Study selection}

All studies were screened by two independent investigators. Disagreements were resolved following discussion with a third investigator followed by reaching a general consensus. The inclusion criteria included studies utilizing blood-based (blood/plasma/serum) circulating tumor cell detection methods in bladder cancer that incorporated a molecular characterization approach. The results of the studies were required to include implications of their work on clinical management.

All conference abstracts, review articles, editorials, comments, letters to the editor and duplicate records were excluded. Studies on urinary biomarkers and non-CTC biomarkers were also excluded from analysis.

\section{Data extraction}

The same authors as above independently extracted data from included articles using a data collection form developed a priori. Data collected included publication details, demographic details, details of CTC detection method, and details of molecular characterization approach. A second investigator confirmed data was extracted accurately.

\section{RESULTS}

\section{Search results}

The PRISMA flowchart is shown in Fig. 1. A total of 102 citations were identified in the database search, 


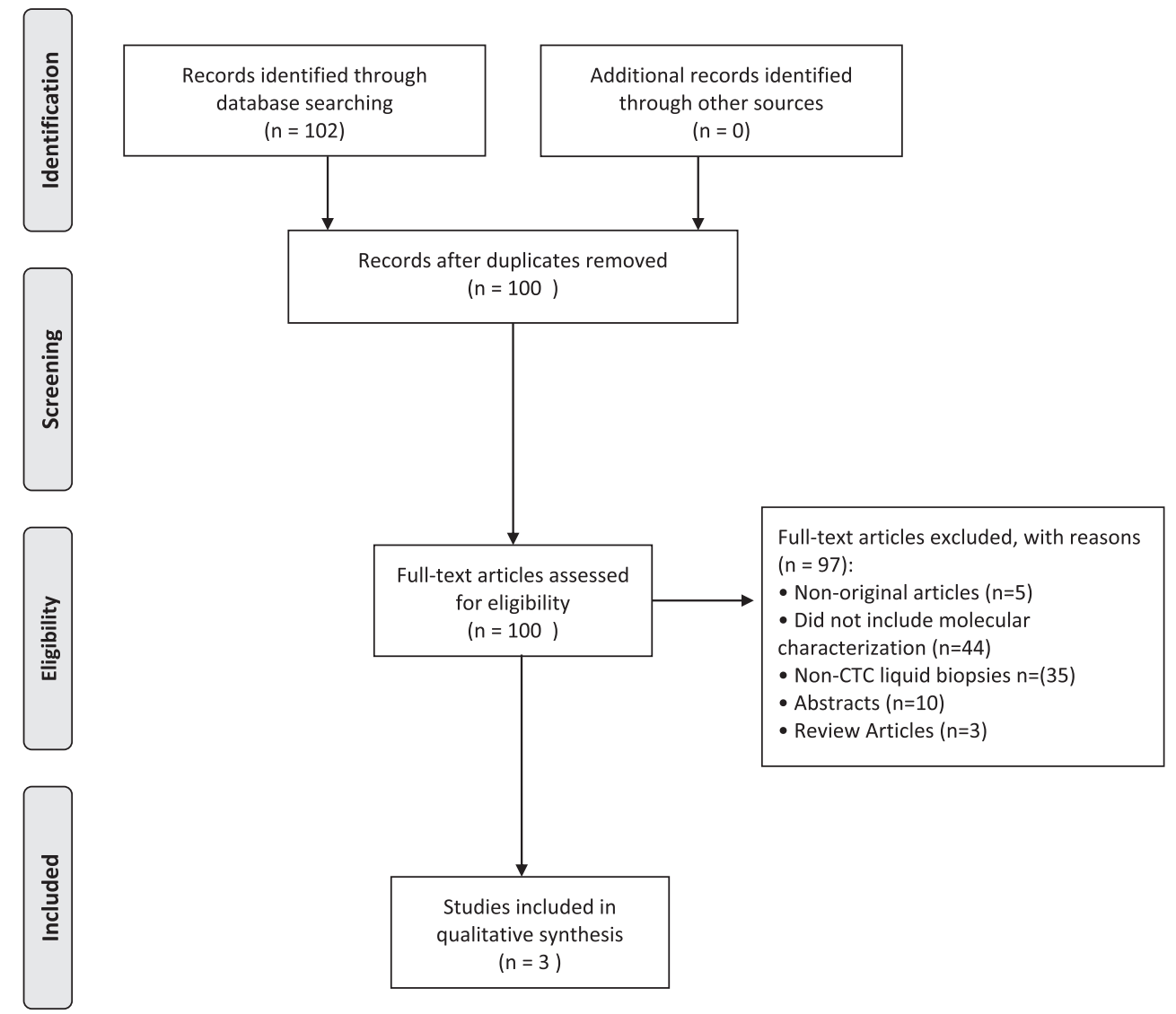

Fig. 1. PRISMA Flow diagram summarizing the systematic review process.

and three studies met the criteria of molecular characterization of CTCs. The studies each interrogated different markers on CTCs including PD-L1, HER2, and sialyl-Tn.

\section{DISCUSSION}

\section{$P D-L 1$}

Bladder cancer tumor cells have been found to express programmed death ligand 1 (PD-L1) on the cell surface [16]. Expression of of PD-L1 on cancer cells helps solid tumors evade immune surveillance and eradication. Discovery of this mechanism led to the development of immune checkpoint inhibitors, such as Atezolizumab. However, biomarkers to predict response to PD-L1 directed therapies are far from established. In bladder cancer, higher PD-L1 expression has been linked to a positive treatment response in select patients [17].
Anatharaman et al. evaluated PD-L1 protein expression on circulating tumor cells (CTCs) isolated from patients with muscle invasive (MIBC) and metastatic bladder cancer ( $\mathrm{mBCa})$, and explored the prognostic value of PD-L1 expression on clinical outcomes. They demonstrated the ability to detect PD-L1 positivity both in cell lines spiked into human blood as well as clinically processed samples [14]. In a small cross sectional cohort they noted that patients with the high PD-L1 positive CTC burden had a shorter overall survival from the time of the CTC draw [14]. However, differences in survival were not statistically significant [14]. Additionally, five evaluable patients received PD-1 targeting therapy after blood was drawn for CTCs, but there was insufficient sample size to assess the prognostic value of PDL1 positive CTCs in patients receiving checkpoint inhibitors [14]. So far this is the only work to apply PD-L1 expression to CTCs, and further prospective cohort studies will be needed to fully confirm its clinical utility. 

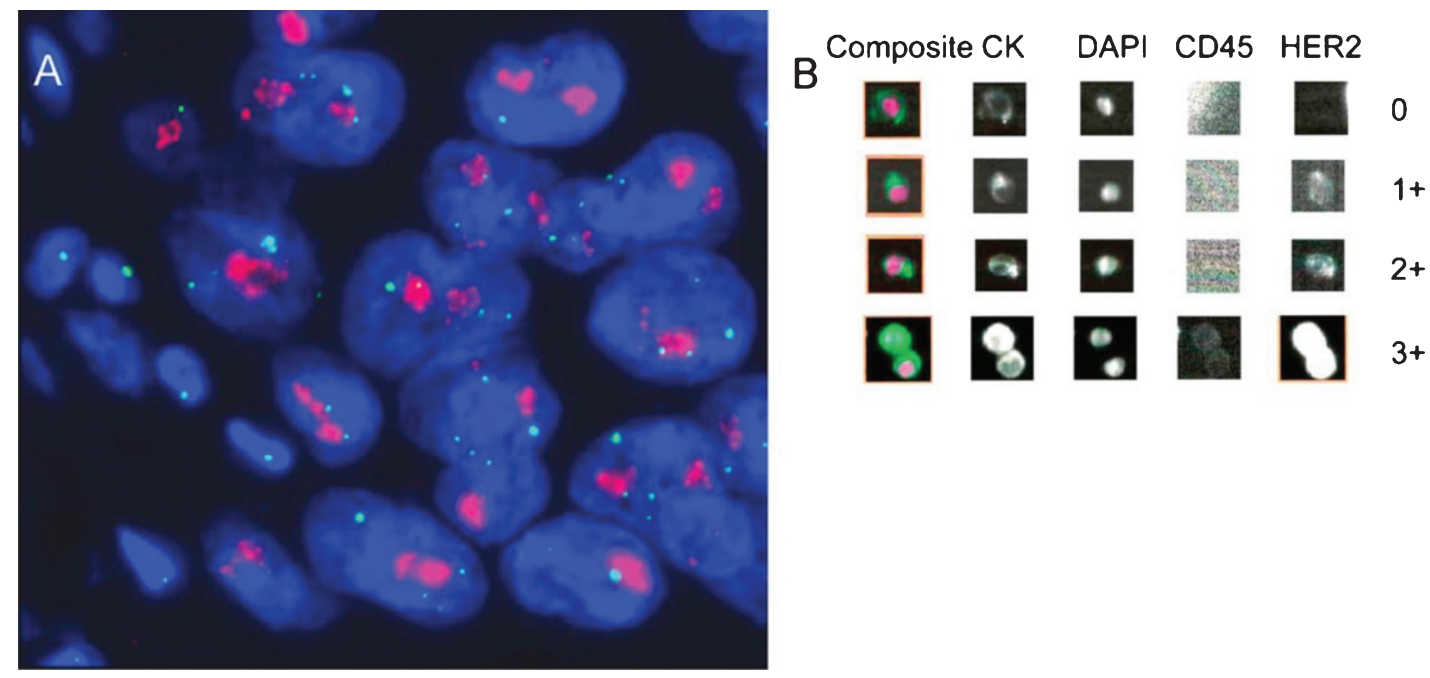

Fig. 2. Determination of human epidermal growth factor receptor 2 (HER2) status by (A) fluorescent in situ hybridization and (B) immunofluorescence using the CellSearch CTC assay system. (A) Primary tumor with strong HER2 gene amplification (clusters of HER2 signals: red; CEP17 signals: green); (B) circulating tumor cells detected with the CellSearch system (0: HER2 negative; 1+: weak; 2+: moderate; 3+: strong intensity of HER2-specific immunofluorescence). Reprinted from European Urology, Volume 61, Issue 4, Rink et. al., Prognostic role and HER2 expression of circulating tumor cells in peripheral blood of patients prior to radical cystectomy: a prospective study., Pages 810-817, Copyright (2012), with permission from Elsevier.

Table 1

Baseline characteristics of included studies

\begin{tabular}{lllll}
\hline $\begin{array}{l}\text { Study } \\
\text { (year) }\end{array}$ & $\begin{array}{l}\text { CTC enrichment } \\
\text { method }\end{array}$ & $\begin{array}{l}\text { Patient } \\
\text { Population }\end{array}$ & $\begin{array}{l}\text { Molecular } \\
\text { Approach }\end{array}$ & $\begin{array}{l}\text { Target antigen / } \\
\text { target gene }\end{array}$ \\
\hline Rink (2012) [13] & CellSearch & MIBC & Immunofluorescence & HER2 \\
Anantharaman (2016) [14] & Epic Sciences & MIBC \& mBCa & Immunofluorescence & PD-L1 \\
Lima (2017) [15] & Microfluidic chip & MIBC & Immunofluorescence & Sialyl-Tn (STn) \\
\hline
\end{tabular}

\section{HER2}

The Human Epidermal Growth Factor Receptor (HER; ErbB) family has been investigated as a target for select patients with bladder cancer given its established role in breast cancer. Human epidermal growth factor receptor 2 (HER2), a tyrosine kinase in the EGFR family, has been found to be associated with biologically aggressive bladder cancer when assessed in the primary tumor [18-20]. There is encouraging early results that that HER2 expression status of bladder cancer cells might predict response to targeted anti-HER2 therapy (Trastuzumab), in select patients [21].

Rink et al. performed a prospective analysis of HER2 expression in CTCs from patients with clinically nonmetastatic bladder cancer. HER 2 expression patterns from CTCs, the primary tumor tissue, and lymph node metastasis were compared for one hundred patients following radical cystectomy. High degrees of concordance were found in the HER2 status of CTCs and the corresponding primary tumors (64\% concordant), and CTCs and lymph node metastases (100\% concordant). CellSearch, an FDA approved CTC detection assay, was used to identify CTCs in this patient population. Use of CellSearch allowed for either in situ hybridization of HER2 DNA or immunofluorescence for HER2 protein (shown in Fig. 2). The rate of HER2 positivity from- CTCs was similar to that reported in studies evaluating HER2 expression in primary tumor tissue from advanced bladder cancer [22]. Of note, $12.5 \%$ of patients with HER2-positive CTCs had primary tumors that were HER2-negative, suggesting that the HER2 status of tumors may evolve, and CTCs may help identify when such transitions occur.

\section{Sialyl-Tn}

Bladder tumors have been shown to overexpress membane glycoproteins, such as thecancerassociated glycoantigen sialyl-Tn (STn) [23-25]. The 
STn antigen is an O-glycan that is associated with high-grade lesions and decreased survival, irrespective of grade and stage of disease [24-27]. STn expression is triggered by high levels of hypoxia, and is involved in modulating protein functions that favor motility, invasion, while promoting and immune escape [24, 26, 28]. These findings suggest that STn may serve as a surrogate biomarker of poor prognosis, especially in patients with advanced disease.

Lima et al. sought to determine the association between STn and disease dissemination by assessing STn expression in circulating tumor cells (CTCs), and in metastastic tumor tissues. They showed that STn expression in both CTC and metastatic lesions was concordant, and provided a strong link between the STn antigen, tumor dissemination, and metastasis. However, these findings need to be confirmed in larger trials with longer followup. In the meantime, STn holds early promise as an effective biomarker for determining tumor aggressiveness and patient stratification.

\section{CONCLUSION}

CTCs hold much promise as a noninvasive strategy for bladder cancer detection and management. The utility of CTCs in precision oncology will benefit from a deliberate focus on molecular information obtained through genotyping, immunohistochemistry, and other approaches. The literature contains three preliminary, but encouraging studies on the potential of molecular characterization of bladder cancer derived CTCs, but there will likely be many more to come as precision medicine becomes integrated into bladder cancer treatment and management.

\section{ETHICAL CONSIDERATIONS}

There are no procedures involving experiments on human or animal subjects.

\section{CONFLICT OF INTEREST}

The authors have no conflict of interest to report.

\section{REFERENCES}

[1] Siegel RL, Miller KD, Jemal A. Cancer statistics, 2018. CA Cancer J Clin. 2018;68(1):7-30. doi:10.3322/caac.21442
[2] Degeorge KC, Holt HR, Hodges SC. Bladder Cancer: Diagnosis and Treatment. 2017;96. www.aafp.org/afp. Accessed March 20, 2019.

[3] Meo A. Di, Bartlett J, Cheng Y, Pasic MD, Yousef GM. Liquid biopsy: A step forward towards precision medicine in urologic malignancies. doi:10.1186/s12943-017-0644-5

[4] Azevedo R, Soares J, Peixoto A, et al. Circulating tumor cells in bladder cancer: Emerging technologies and clinical implications foreseeing precision oncology. Urol Oncol Semin Orig Investig. 2018;36:221-36. doi:10.1016/j.urolonc.2018.02.004

[5] Zhang Z, Fan W, Deng Q, et al. The prognostic and diagnostic value of circulating tumor cells in bladder cancer and upper tract urothelial carcinoma: A meta-analysis of 30 published studies. Oncotarget. 2017;8(35):59527-38. doi:10.18632/oncotarget.18521

[6] Zhang L, Riethdorf S, Wu G, et al. Meta-Analysis of the Prognostic Value of Circulating Tumor Cells in Breast Cancer. Clin Cancer Res. 2012;18(20):5701-10. doi:10.1158/1078-0432.CCR-12-1587

[7] Hou J-M, Krebs MG, Lancashire L, et al. Clinical significance and molecular characteristics of circulating tumor cells and circulating tumor microemboli in patients with small-cell lung cancer. J Clin Oncol. 2012;30(5):525-32. doi: $10.1200 / J C O .2010 .33 .3716$

[8] Huang X, Gao P, Song Y, et al. Relationship between Circulating Tumor Cells and Tumor Response in Colorectal Cancer Patients Treated with Chemotherapy: A Meta-Analysis.; 2014. doi:10.1186/1471-2407-14-976

[9] Zhang W-Q, Li T, Li H. Efficacy of EGFR tyrosine kinase inhibitors in non-small-cell lung cancer patients with/without EGFR-mutation: Evidence based on recent phase III randomized trials. Med Sci Monit. 2014;20:266676. doi:10.12659/MSM. 892476

[10] Wilson FR, Coombes ME, Brezden-Masley C, et al. Herceptin ${ }^{\circledR}$ (trastuzumab) in HER2-positive early breast cancer: A systematic review and cumulative network metaanalysis. Syst Rev. 2018;7(1):191. doi:10.1186/s13643018-0854-y

[11] Antonia SJ, Villegas A, Daniel D, et al. Durvalumab after Chemoradiotherapy in Stage III Non-Small-Cell Lung Cancer. N Engl J Med. 2017;377(20):1919-29. doi:10.1056/NEJMoa1709937

[12] Petrylak DP. Immunotherapy: The Wave of the Future in Bladder Cancer? Clin Genitourin Cancer. 2017. doi:10.1016/j.clgc.2017.05.025

[13] Rink M, Chun FK, Dahlem R, et al. Prognostic Role and HER2 Expression of Circulating Tumor Cells in Peripheral Blood of Patients Prior to Radical Cystectomy: A Prospective Study. doi:10.1016/j.eururo.2012.01.017

[14] Anantharaman A, Friedlander T, Lu D, et al. Programmed death-ligand 1 (PD-L1) characterization of circulating tumor cells (CTCs) in muscle invasive and metastatic bladder cancer patients. BMC Cancer. 2016;16. doi:10.1186/s12885-016-2758-3

[15] Lima L, Neves M, Oliveira MI, et al. Sialyl-Tn identifies muscle-invasive bladder cancer basal and luminal subtypes facing decreased survival, being expressed by circulating tumor cells and metastases. Urol Oncol Semin Orig Investig. 2017;35:675.e1-675.e8. doi:10.1016/j.urolonc.2017. 08.012

[16] Davarpanah NN, Yuno A, Trepel JB, Apolo AB. Immunotherapy: A new treatment paradigm in bladder cancer. Curr Opin Oncol. 2017;29(3):184. doi:10.1097/CCO.00000 00000000366 
[17] Rosenberg JE, Hoffman-Censits J, Powles T, et al. Atezolizumab in patients with locally advanced and metastatic urothelial carcinoma who have progressed following treatment with platinum-based chemotherapy: A single-arm, multicentre, phase 2 trial. Lancet (London, England). 2016;387(10031):1909-20. doi:10.1016/S01406736(16)00561-4

[18] Gandour-Edwards R, Lara PN, Folkins AK, et al. DoesHER2/neu expression provide prognostic information in patients with advanced urothelial carcinoma? Cancer. 2002;95(5):1009-15. doi:10.1002/cncr.10808

[19] Bolenz C, Shariat SF, Karakiewicz PI, et al. Human epidermal growth factor receptor 2 expression status provides independent prognostic information in patients with urothelial carcinoma of the urinary bladder. BJU Int. 2010;106(8):1216-22. doi:10.1111/j.1464410X.2009.09190.x

[20] Laé M, Couturier J, Oudard S, Radvanyi F, Beuzeboc P, Vieillefond \& A. Assessing HER2 gene amplification as a potential target for therapy in invasive urothelial bladder cancer with a standardized methodology: Results in 1005 patients. Ann Oncol. 2010;21:815-9. doi:10.1093/ annonc/mdp488

[21] Hussain MHA, MacVicar GR, Petrylak DP, et al. Trastuzumab, paclitaxel, carboplatin, and gemcitabine in advanced human epidermal growth factor receptor- $2 /$ neupositive urothelial carcinoma: Results of a multicenter phase II National Cancer Institute trial. J Clin Oncol. 2007;25(16):2218-24. doi:10.1200/JCO.2006.08.0994

[22] Fleischmann A, Rotzer D, Seiler R, Studer UE, Thalmann GN. Her2 Amplification is Significantly More Frequent in Lymph Node Metastases From Urothelial Bladder Cancer Than in the Primary Tumours. doi:10.1016/ j.eururo.2011.05.035
[23] Bernardo C, Costa C, Amaro T, et al. Patient-derived sialyl-Tn-positive invasive bladder cancer xenografts in nude mice: An exploratory model study. Anticancer Res. 2014;34(2):735-44. http://www.ncbi.nlm.nih. gov/pubmed/24511007. Accessed March 20, 2019.

[24] Ferreira JA, Videira PA, Lima L, et al. Overexpression of tumour-associated carbohydrate antigen sialyl-Tn in advanced bladder tumours. Mol Oncol. 2013;7(3):719-31. doi:10.1016/J.MOLONC.2013.03.001

[25] Costa C, Pereira S, Lima L, et al. Abnormal Protein Glycosylation and Activated PI3K/Akt/mTOR Pathway: Role in Bladder Cancer Prognosis and Targeted Therapeutics. Real FX, ed. PLoS One. 2015;10(11):e0141253. doi:10.1371/journal.pone.0141253

[26] Peixoto A, Fernandes E, Gaiteiro C, et al. Hypoxia enhances the malignant nature of bladder cancer cells and concomitantly antagonizes protein $O$-glycosylation extension. Oncotarget. 2016;7(39):63138-57. doi:10.18632/onco target. 11257

[27] Cotton S, Azevedo R, Gaiteiro C, et al. Targeted $O$ -glycoproteomics explored increased sialylation and identified MUC16 as a poor prognosis biomarker in advancedstage bladder tumours. Mol Oncol. 2017;11(8):895-912. doi:10.1002/1878-0261.12035

[28] Carrascal MA, Severino PF, Guadalupe Cabral M, et al. Sialyl Tn-expressing bladder cancer cells induce a tolerogenic phenotype in innate and adaptive immune cells. Mol Oncol. 2014;8(3):753-65. doi:10.1016/J.MOLONC.2014.02.008 